\title{
Front Propagation in Stochastic Neural Fields
}

In this talk we will introduce a phenomenologically motivated rigorous mathematical framework for the analysis of stochastic neural field equations with spatially extended additive noise. It is well known that in the deterministic case neural field equations allow for the existence of monotone travelling-wave solutions. As described in [1], additional extrinsic stochastic forcing terms result in two distinct phenomena: perturbations of the front shape as well as a horizontal displacement of the wave profile from its uniformly translating position. The mathematical framework we develop captures these effects and allows us to prove new stability results. In the second part of the talk we will then consider existence and uniqueness of solutions in the case where the nonlinearity of the equation is discontinuous.

\section{References}

[1] P.C. Bressloff, M.A. Webber. Front Propagation in Stochastic Neural Fields, SIAM J. Applied Dynamical Systems 11 (2) pp. 708-740, 2012. 